\title{
la dimension éducation dans le traitement odonto-stomatologique
}

RÉSUMÉ L'éducation du patient devient une dimension incontournable des thérapeutiques modernes. Elle permet une meilleure relation de traitement, agit de façon indirecte sur la motivation du patient et sur des facteurs biologiques en positif. Elle vise un objectif de santé globale en mettant en action un comportement positif de conscience du patient sur la prise en charge autonome de sa santé. Actuellement, la méthode pédagogique utilisée est l'explication, méthode inefficace qui doit être remplacée par les résultats des recherches de spécialistes en éducation de la santé.

Marc-Gérald CHOUKROUN

Spécialiste qualifié en O.D.F.,

51, avenue Henri Ginoux,

92120 Montrouge.

\section{MOTS CLÉS}

éducation $d u$ patient

relation praticien/patient

psychologie 


\section{introduction}

C

haque fois que le praticien doit énoncer une information au patient, que ce soit relatif au diagnostic, au traitement proposé, ou bien à des ordonnances ou des prescriptions diverses, des consignes post-opératoires et en particulier sur l'hygiène buccodentaire, il devient malgré lui pédagogue. Quelles sont les techniques psycho-pédagogiques actuellement en usage ?

L'explication est la méthode la plus largement utilisée, parfois ce sont certains écrits, éventuellement des programmations vidéo.

Or nous savons aujourd'hui que nous retenons :

$-10 \%$ de ce que nous lisons,

- $20 \%$ de ce que nous entendons,

$-30 \%$ de ce que nous voyons.

Ainsi l'explication se présente comme une méthode peu efficace.

Certains auteurs se sont penchés sur la question de l'éducation en santé, et il semble important que les professions de santé en soient informées.

L'apprentissage se fonde sur les méthodes décrites par Deccache et d'Yvernois.

Voici la définition d'Alain Deccache:
«C'est un processus étape par étape intégré dans la démarche de soins ».

La condition essentielle d'une démarche pédagogique est la disponibilité de l'enseignant et de l'enseigné. Citons, pour exemple, la tentative malheureuse de ce confrère : la patiente demande au praticien comment éviter les caries chez sa jeune fille qui présente une atteinte polycarieuse. Le praticien lui explique qu'il faut agir sur plusieurs niveaux et lui dresse une liste sur ordonnance des actions à mener :

1 - soins et détartrage ;

2 - 15 jours de bains de bouche à la chlorexidrine ;

3 - équilibrer le régime alimentaire ;

4 -manger des chewing-gums sans sucre après les repas ;

5 - bains de bouche fluorés le soir avant le coucher sans rinçage ;

6 - utiliser un dentifrice au fluor ;

7 - effectuer régulièrement des contrôles dentaires et les soins appropriés.

La réponse de la patiente a été faite par courrier :

«Monsieur,

Je vous tiens informé de ce que je préfère attendre le vaccin anti-caries.

Veuillez agréer, Monsieur, l'expression de mes salutations distinguées».

\section{la pédagogie médicale}

Ce qui perturbe d'emblée la relation pédagogique est le fait que le praticien ne s'inquiète jamais du savoir $d u$ patient. Dans les années 80 , je suis allé dans les écoles pour expliquer aux enfants l'origine des maladies buccodentaires et leur prévention. J'ai été surpris de constater que des élèves 
de CM1 et de CM2 possédaient un niveau de connaissance très étayé. Ils savaient que la carie provenait des sucres, des microbes, que les dents détériorées devaient être reconstruites avec des "plombages», des couronnes, des bridges et qu'il était nécessaire de se brosser les dents après chaque repas. En leur répétant ces éléments de savoir, qu'aurais-je fait sinon de les ennuyer et de les culpabiliser? C'est pourquoi le temps éducatif était consacré à la découverte de la cavité buccale : les zones, les sensations, les fonctions, afin de les familiariser avec une partie de leur corps qui restait mystérieuse. L'objectif pédagogique devenait alors : "Je connais ma bouche, je n'en ai pas peur, je prends plaisir à la soigner». C'est pourquoi Deccache parle de commencer par le diagnostic éducatif pour déterminer la compétence du patient. Ensuite, il faut s'assurer de la disponibilité du malade : ne pas enseigner n'importe quoi à n'importe quel moment. Le comportement visé doit être le sujet d'un programme pédagogique.
La répétition est le fondement de toute pédagogie. Celle-ci est un art. Le praticien doit répéter ses informations sans en avoir l'air. Pour cela, il doit utiliser toutes formes de supports. Ses auxiliaires doivent eux (ou elles) aussi faire redondance. Il importe peu que le message soit différent d'un support à l'autre, sa variété permet d'étayer sa complexité et le rend plus attrayant. Du praticien à l'assistante, de l'assistante au CD ROM ou au dépliant, les messages doivent circuler de façon incessante tout au long du traitement. Il ne faut en aucun cas se contenter de l'énoncer une fois pour toutes. La maladresse provient du praticien qui répète malgré lui et exprime un agacement.

La répétition doit être un acte automatique, neutre et bienveillant.

En additionnant l'explication, la lecture et la vidéo nous passons donc à $60 \%$ de rétention.

Mais ce n'est pas la technique la plus intéressante et la plus rapide.

\section{la pédagogie des informations}

Il existe deux types d'informations que le praticien doit délivrer pédagogiquement à son patient :

L'information sécuritaire est l'information incontournable pour exercer la thérapeutique, elle doit être détachée, simple et ne peut être confondue avec tout autre information. Ce sont les prescriptions, en précisant que les prescriptions de l'art médical ne se limitent pas à une ordonnance. Il faut y inclure les conditions de prise des médicaments, l'attitude à avoir face à une difficulté, l'hygiène de vie, les obligations du patient dans son traitement.

Mais isolée, elle n'est pas motivante, ce ne sont que des contraintes comme seule réponse à une personne qui souffre et s'inquiète.

Les informations générales ne sont là que pour donner un cadre idéologique et pédagogique positif à la relation. Il faut lui montrer que l'on connaît son problème médical et que la science pré- 
sente des solutions. Il nous paraît également important devant la soif de guérison de certains patients, de leur rappeler l'histoire de la médecine et de leur montrer que les nouvelles solutions représentent des progrès considérables : Louis XIV à trente ans n'avait plus de dents au maxillaire!

\section{la technique par la maïeutique}

Notre expérience auprès des professionnels de santé montre qu'ils agissent de façon systématique par le modèle explicatif. Or le malade n'entend plus rien après trois minutes d'attention. La technique par explication implique deux impératifs : elle doit être courte, elle ne doit intervenir qu'après avoir posé au patient certaines questions qui visent à l'impliquer dans les réponses.

La meilleure méthode est celle de la maïeutique. Elle vise à faire parler le patient en l'aidant à découvrir par luimême les explications que l'on désire transmettre. Or la statistique nous montre sa supériorité, ce qui est dit par le patient est retenu à $80 \%$.

Le mérite en revient à SOCRATE d'être l'inventeur de cette pédagogie. Maïos en grec signifie l'accouchement. L'art consiste à faire accoucher l'élève des éléments de savoir du maître. Rappelons l'exemple de l'hygiène dentaire ( in «Abrégé de psychologie» de l'auteur). C'est le Docteur Tom qui s'entretient avec un jeune patient.

Dr Tom : - Pourquoi doit-on se brosser les dents?

François : - Parce que l'on mange...

Dr Tom : - Et alors ?

François: - Alors cela attire les microbes.

Dr Tom : - Et que font les microbes?
François : - Ils font des caries...

Continuons la conversation suggérée dans cet exemple.

Dr Tom : - Comment les éviter ?

François : - En les brossant.

Dr Tom : - Quand?

François : - Quand on a mangé.

Dr Tom : - C'est-à-dire ?

François : - Ben ... après les repas.

Dr Tom : - Comment les brosser ?

François : - Je ne sais pas...

Dr Tom : - Que doit-on enlever ?

François : - Les microbes.

Dr Tom : - Où sont-ils ?

François : - Sur les dents?

Dr Tom : - Tu les vois?

François : - Non.

Dr Tom : - Alors comment faire ?

François: - Là où sont les aliments...?

Dr Tom : - C'est cela, et quel geste ferons-nous?

François: - Je ne sais pas.

Dr Tom : - Je vais t'expliquer...

Ainsi, de question en question, la technique consiste à guider l'élève vers les réponses qu'il découvrira par lui-même. C'est à ce moment seulement que peut intervenir le soignant. Il propose une brosse à dents au patient ainsi qu'un miroir et joint le geste à la parole : 
«Tu poses ta brosse au milieu de l'arcade. Les poils vers le haut pour les dents du haut et vers le bas pour les dents $\mathrm{du}$ bas. Tu progresses vers le fond en faisant un petit massage, comme la fumée d'un train. Tu fais le tour derrière la dernière molaire pour longer l'intérieur et revenir finalement au point de départ».

Des explications simples, répondant à l'interrogation du patient.

Il est préférable de compléter les explications suivantes dans une séance ultérieure, de sorte que le patient puisse assimiler les premières. La pédagogie s'arrête là où les aptitudes d'assimilation du patient s'arrêtent. Et ces limites sont très vites atteintes!

Cette technique est utilisable dans toutes les situations. Par exemple, pour présenter au patient une dent de sagesse incluse, il est possible de montrer la radio au patient et de lui demander :

Le praticien : - Que voyez-vous ?

Le patient : - Mes dents?

Le praticien : - Oui et là (le praticien indique la 8 avec un stylo)

$$
\begin{aligned}
& \text { Le patient: - C'est ma dent de } \\
& \text { sagesse? }
\end{aligned}
$$

Le praticien : - Oui et comment est-elle?

Le patient : - Elle est dans l'os?

Le praticien : - Et alors?

Le patient : - Elle est bloquée?

Le praticien: - C'est exact, elle est incluse, que faire?

Le patient: - Il faut ouvrir la gencive.

Le praticien : - Et encore ?

Le patient : - Et dégager l'os?

Le praticien : - C'est exact...

Le patient : - C'est une opération?

Le praticien : - Une petite chirurgie...

Ainsi, de question en question, il est possible de tout faire dire au patient qui s'implique, comprend nos gestes, les difficultés et la compétence du praticien. Nous serons gagnants sur tous les tableaux.

Tous les praticiens que nous avons formés à cette technique en sont convaincus et ne peuvent plus concevoir d'agir autrement.

\section{les autres techniques}

\section{- la démonstration}

Elle consiste à effectuer l'exercice de brossage sur un modèle en résine avec une brosse à dents. Le patient sera invité ensuite à effectuer le geste sur ses dents. L'éducateur relève alors les anomalies et les lui corrige. La démonstration peut être utilisée pour tout type de manipulation. Elle sera bienvenue pour déposer et poser un appareil amovible sur un moulage en plâtre. Elle peut également s'envisager pour de nombreux cas en O.D.F. et en parodontologie.

\section{- I'atelier}

On réunit sur une table divers objets qui attirent la curiosité du sujet et permettent d'illustrer des explications telles que brosses variées, révélateur de 
plaque, fil de soie, modèles de démonstration, images, photos de gingivites, des dessins,... La technique consiste à laisser le patient choisir les objets qui l'intriguent et suscitent des questions. On permet au patient de verbaliser ses connaissances, ses interrogations et on structure son savoir. "À quoi sert cet objet ?» «Pourquoi ?...». L'atelier permet de travailler en interactivité.

Il est possible d'utiliser des supports d'informations comme un prospectus, une cassette vidéo, un CD ROM.

\section{- l'apprentissage vicariant}

Cela consiste à demander à un autre patient qui a bien assimilé l'acte, de le présenter, de parler de sa propre expérience. Cette technique est très favorable à la pose d'un appareillage.

\section{- le soutien}

Il utilise l'aide d'un proche : faire participer la maman au brossage des dents de l'enfant pour un sujet très jeune, ou sur elle-même.

On peut organiser un groupe de réflexion entre des soignants et plusieurs patients sur un thème de réflexion :
Par exemple, sur le thème du choix des brosses et $d u$ dentifrice. Ou encore : comment gérer les trois premiers jours après la pose d'un appareil multiattache.

\section{le dessin}

C'est une technique indirecte de démonstration. Par son aspect projectif (le patient dessine ce qui pose problème), le dessin favorise une verbalisation des inquiétudes qui sont parfois difficiles à exprimer en face à face. Au travers des dessins, on expliquera comment sont les dents, les gencives, la prothèse. L'éducateur apporte lui-même les compléments graphiques.

L'accumulation de ces techniques par leurs différents aspects et leur redondance engendre la compréhension et la mémorisation. Utilisées par plusieurs personnes, elles favorisent chez le patient l'appréhension d'une équipe de soins. Leur temps peut varier selon les exigences $d u$ soin : de quelques minutes à une demi-heure. Elles doivent faire l'objet, comme l'explique Alain Deccache, d'un projet pédagogique. L'équipe soignante doit inclure dans son programme de traitement des espaces pédagogiques : prévoir leur contenu et leur durée.

\section{la disponibilité}

\section{Pour apprendre il faut être disposé à} apprendre.

Il faut donc prévoir de petites étapes selon les informations qui doivent être assimilées, qui se suivent selon un programme pédagogique préétabli par l'équipe soignante. Si chaque étape est simple et réussie par les patients, cela représentera la meilleure motivation pour encourager le patient à plus 
apprendre et à se sentir en confiance avec les soignants.

La sensibilisation consiste à éveiller la curiosité du patient: "Désirez-vous savoir si votre hygiène est correcte ?»

"Désirez-vous savoir comment on déchiffre cette radiographie ?»

\section{Les quatre conditions :}

«Pas n'importe où, pas n'importe quand, pas n'importe comment, pas n'importe qui».

Les erreurs pédagogiques peuvent, la plupart du temps, être attribuées à la transgression de cette règle simple.

Le praticien engage une conversation qui représente une véritable information au moment de quitter le patient, en ouvrant la porte : «Et si vous avez trop mal, prenez un antalgique...». Il s'agit d'une prescription : c'est donc une information médicale. On doit s'assurer que le patient en possède à son domicile, sinon il faut établir une ordonnance. Il faut s'assurer qu'il n'y a pas de contre-indications : donc faire un interrogatoire. Le seuil de la porte n'est pas le lieu d'un acte : pas n'importe où.

Le praticien donne une leçon d'hygiène à un enfant sur le ton de la culpabilisation : "Continue comme cela et tu auras toutes les dents cariées...». Cette réflexion répond en principe à une explication sur le danger d'une mauvaise hygiène et ses conséquences. C'est un discours scientifique et préventif. Dans cet exemple, le praticien n'est pas crédible, les informations sont absentes et leur lien inexistant.
La culpabilité n'a jamais déclenché un comportement de prévention: pas n'importe comment.

Voici maintenant que le praticien a passé un long moment d'intervention et soudain, il s'aperçoit que l'hygiène n'est pas suffisante. "Il faut mieux vous laver les dents, n'est-ce pas ?». Cette phrase ne peut constituer une leçon d'hygiène dentaire. Le praticien doit prévoir une séance à cet effet. "Je vois que votre hygiène n'est pas suffisante, nous allons prévoir un temps pour en parler. En attendant, tentez de réfléchir à cette question pour que nous ayons une bonne conversation tous les deux»: pas n'importe quand.

Le praticien s'adresse à la maman: «Madame, votre fils doit faire attention à son hygiène...». Si c'est le fils qui doit être vigilant, pourquoi s'adresser à la mère ? Si la mère à un rôle à jouer, quel est-il ? Voilà encore un raccourci de pédagogie qui laisse toutes les parties dans l'embarras. Il vaudrait mieux dire :

«Paul, ton hygiène n'est pas suffisante, il serait bon que tu reprennes les éléments que nous t'avons enseignés, s'il existe une difficulté, il faut me le dire pour que je puisse t'aider.

Madame, serait-il possible d'observer son comportement le soir à la salle de bains et de nous informer la prochaine fois des difficultés qu'il rencontre ?».

Nous nous situons alors dans un rapport pédagogique de participation et d'implication. Pas n'importe qui. 


\section{l'évaluation}

Le concept d'évaluation est inhérent au domaine de la pédagogie. Elle fonde sa valeur et son efficacité. Loin de sanctionner l'élève, elle sanctionne le maître et le met face à ses lacunes. L'évaluation négative est une recherche sur les outils qui ont mal fonctionné, l'évaluation positive est la garantie $d u$ bon fonctionnement de ces outils. Elle n'est pas spécifique à la pédagogie, elle est le corollaire de tout travail quel qu'il soit. L'évaluation pose le problème des critères de jugement. Tout ce qui concerne les objectifs de traitement doit subir cet effort de déterminer des critères d'évaluation.

\section{- évaluation d'un apprentissage}

En ce qui concerne l'hygiène, l'affaire est simple: c'est l'absence de plaque dentaire et de gingivite.

Cependant, il ne faut pas confondre l'objectif avec un résultat immédiat. L'apprentissage doit passer par une «négociation».

Dans l'hygiène bucco-dentaire par exemple, il sera bon de fixer avec le patient des étapes : si chaque étape est simple à réaliser, le patient sera encouragé, le praticien également et d'étape en étape, il sera aisé d'atteindre les objectifs.

$\mathrm{Au}$ contraire, dans la réalité clinique, nous constatons que certains praticiens exigent de leur patient de réaliser en une seule étape les objectifs. Cette erreur pédagogique entraîne une démotivation et du patient et $\mathrm{du}$ praticien, désespoir qui peut dans certains cas se répercuter sur le traitement lui-même.

\section{- évaluation des informations}

Dans toutes les informations, l'évaluation doit être effectuée en demandant au patient de reformuler ce qu'il a retenu.

Cette attitude doit être automatique au cours du bref entretien qui a eu lieu, car nous sommes bien conscients que le temps de la consultation ne doit jamais être prolongé inutilement.

Cependant, nous constatons que les complications des traitements, les demandes réitérées de patients inquiets ou insatisfaits, les revendications de certains, prolongent nos consultations de façon stressante et sans résultat.

\section{évaluation} du vécu du traitement

Enfin, le praticien devra évaluer incessamment le bien-être du patient dans son vécu du traitement. La réussite clinique est un traitement qui laisse derrière lui une expérience positive. Notre suivi clinique montre, à l'évidence, que le traitement aussi pénible soit-il de par ses contraintes ou ses douleurs, peut être sanctionné par la satisfaction du patient si la pédagogie a bien réussi.

Les critères psychologiques et éducatifs seront de nouveaux critères pour le professionnel de santé, comme l'ont montré de nombreux articles. 


\section{objectifs de l'éducation du patient}

Outre la bonne conduite du traitement, l'adhésion et la participation $d u$ patient, l'objectif éducatif est aujourd'hui modélisé par le projet de santé globale.

Par le biais de tout traitement, le patient doit apprendre à se soigner, à savoir prendre sa place face aux professionnels de santé pour obtenir le meilleur de leur compétence. Il doit dédramatiser son corps en le démystifiant afin d'en prendre soin d'une façon autonome et préventive.

Nous avons connu par le passé, et elles existent encore, des attitudes réfractaires aux soins par certains patients qui, faute d'éducation et de représentation positive de la santé, ne fréquentaient nos consultations que dans des situations d'urgence.

\section{conclusion}

Le praticien est sollicité en permanence par les méthodes psychopédagogiques. Il n'en a pas toujours conscience et souvent il les ignore. Comment s'étonner alors de l'inefficacité des résultats ? Il faut apprendre à apprendre.
L'éducation du patient n'est pas seulement un cadre intellectuel de la relation médecin-malade, elle représente par une collatérale la méthode qui permet au patient de participer positivement à son traitement et de favoriser ses facteurs biologiques.

\section{ouvrages à consulter}

Aumont B, Mesnier PM.

L'acte d'apprendre.

Paris : P.U.F.

(coll. Pédagogie d'Aujourd'hui), 1995:282 p.

Béry A.

Le contrat de soins.

Paris : S.I.D., 1997.

Bury AJ.

Éducation pour la santé, concepts, enjeux, planification. Bruxelles : De Boeck Université, 1988:234

Choukroun MG.

La mise en acte du traitement. Paris : S.I.D., 2004:250 p.
Choukroun MG

Les échecs relationnels en orthodontie.

Rev Orthop Dento Faciale 2000;34:313-323.

Choukroun MG

Abrégé de psychologie

à l'usage des praticiens.

Paris : S.I.D., 1997:226 p.

Deccache A, Lavendhomme E. Information et éducation du patient : des fondements aux méthodes.

Bruxelles : De Boeck Université (coll. Savoir et Santé),

1989:239 p
Gagnayre R, d'Ivernois JF. Apprendre à éduquer le patient, approche pédagogique. Paris : Vigot (coll. Education du patient), 1995:177 p.

Gordon T, Edwards S.

Communiquer avec ses patients.

Paris : Logiques éd.

(Le Jour éd. française), 1997:315 p.

Schoonbroodt C, Coppé M.

Guide pratique d'éducation pour la santé.

Bruxelles : Deboeck Université, 1992:299 p. 


\section{Education field in the odonto-stomatological treatment}

M.-G. CHOUKROUN

Patient education has become imperative to be introduced into the modern treatment plannings. It allows a better relationship beetwen the practitioner and his patient, and indirectly acts upon motivation and biological factors to help care. It also represents an essential goal as «holly health» as well as looking for a bet- ter consciousness by the patient about its own health.

At the present time, the most common used method is under explanations which efficiency is negative. This must be now replaced by results of research made about the health education field.

keywords: patient education, practitioner-patient relationship, psychology.

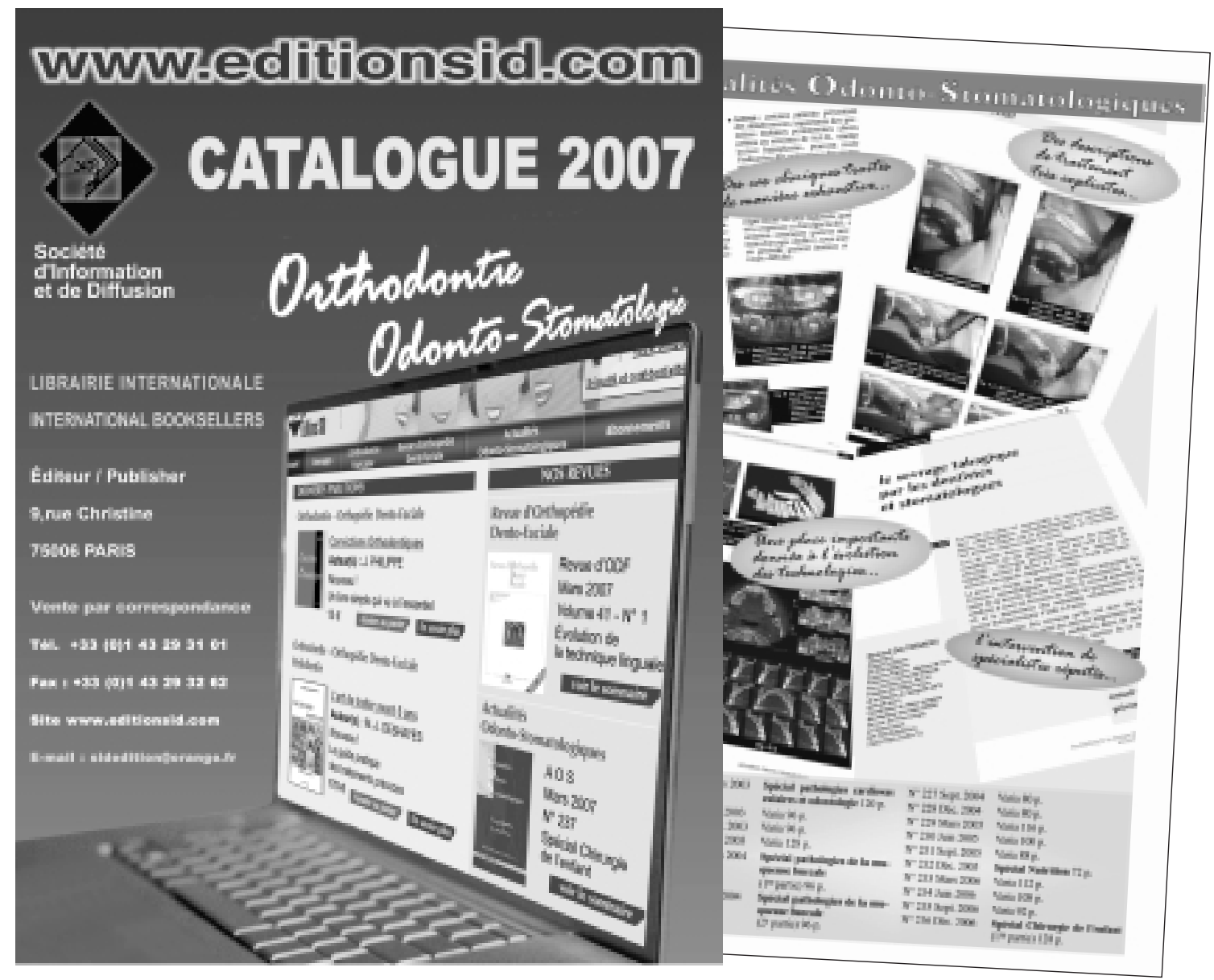

